\title{
Current perspectives on statins as potential anti-cancer therapeutics: clinical outcomes and underlying molecular mechanisms
}

\author{
Ali Fatehi Hassanabad \\ Cumming School of Medicine, University of Calgary, Calgary, Alberta, Canada \\ Correspondence to: Ali Fatehi Hassanabad. Room 880, Main Building, $140329^{\text {th }}$ Street NW, Foothills Medical Centre, Cumming School of Medicine, \\ University of Calgary, Calgary, AB, T2N 2T9, Canada. Email: ali.fatehihassanabad@ahs.ca.
}

\begin{abstract}
Statins have been shown to inhibit cell proliferation in vitro and tumor growth in animal models. Various studies have also shown a decreased cancer-specific mortality rate in patients who were prescribed these medications. Statins inhibit 3-hydroxy-3-methylglutaryl-CoA reductase (HMGCR), the rate-limiting enzyme of the mevalonate pathway. Statins induce tumour-specific apoptosis through mitochondrial apoptotic signaling pathways, which are activated by the suppression of mevalonate or geranylgeranyl pyrophosphate (GGPP) biosynthesis. However, there is no consensus on the molecular targets of statins for their anti-cancer effects. Several studies have been conducted to further assess the association between statin use and mortality in different types of cancer. In this review, current perspectives on clinical significance of statins in prevention and treatment of various types of cancers and proposed mechanisms are discussed.
\end{abstract}

Keywords: Statins; cancer; clinical outcomes; cancer therapeutics; cellular and molecular cancer

Submitted Jul 29, 2019. Accepted for publication Aug 21, 2019.

doi: 10.21037/tlcr.2019.09.08

View this article at: http://dx.doi.org/10.21037/tlcr.2019.09.08

\section{Introduction}

According to the World Health Organization (WHO) cancer is a generic term for a large group of diseases that can affect any part of the body, and metastases are a major cause of death from cancer. Based on WHO statistics, cancer is the second leading cause of death globally. The economic burden of cancer is significant as its estimated total annual cost has been over US\$ 1.1 trillion over the past decade. The health and socioeconomic implications of cancer underscore an urgent need for identifying and applying successful preventative and therapeutic options (1).

Chemotherapy is a common approach to cancer management, which is currently used in a curative, palliative, or adjuvant capacity. Several classes of chemotherapeutic medications have been used clinically, including DNA alkylating agents, platinating agents, and antimetabolites. Although these drugs have had varying degrees of success, they also possess significant undesired side effects resulting in their diminished utility. Further complicating matters, in cases where these drugs initially prove efficacious, tumours can develop mechanisms of drug resistance. Such mechanisms include increasing the expression of multidrug efflux pumps, altering the expression of the drug's target, and upregulating survival pathways. To resolve these issues and improve treatment outcomes, researchers are keen on developing tumour-specific agents.

Many current chemotherapeutics have been shown to kill tumour cells by inducing apoptosis (2). Apoptosis which is a genetically programmed cell death is executed by two major pathways: the extrinsic pathway and the intrinsic pathway. Apoptotic pathways require an array of functional interactions that would ensure effective regulation of programmed cell death. A tumour could acquire a host of mutations or alterations in order to evade apoptosis. Although escaping programmed cell death is a pivotal characteristic for tumourigenesis, it does not seem to be a general response to all apoptotic stimuli. Paradoxically, 


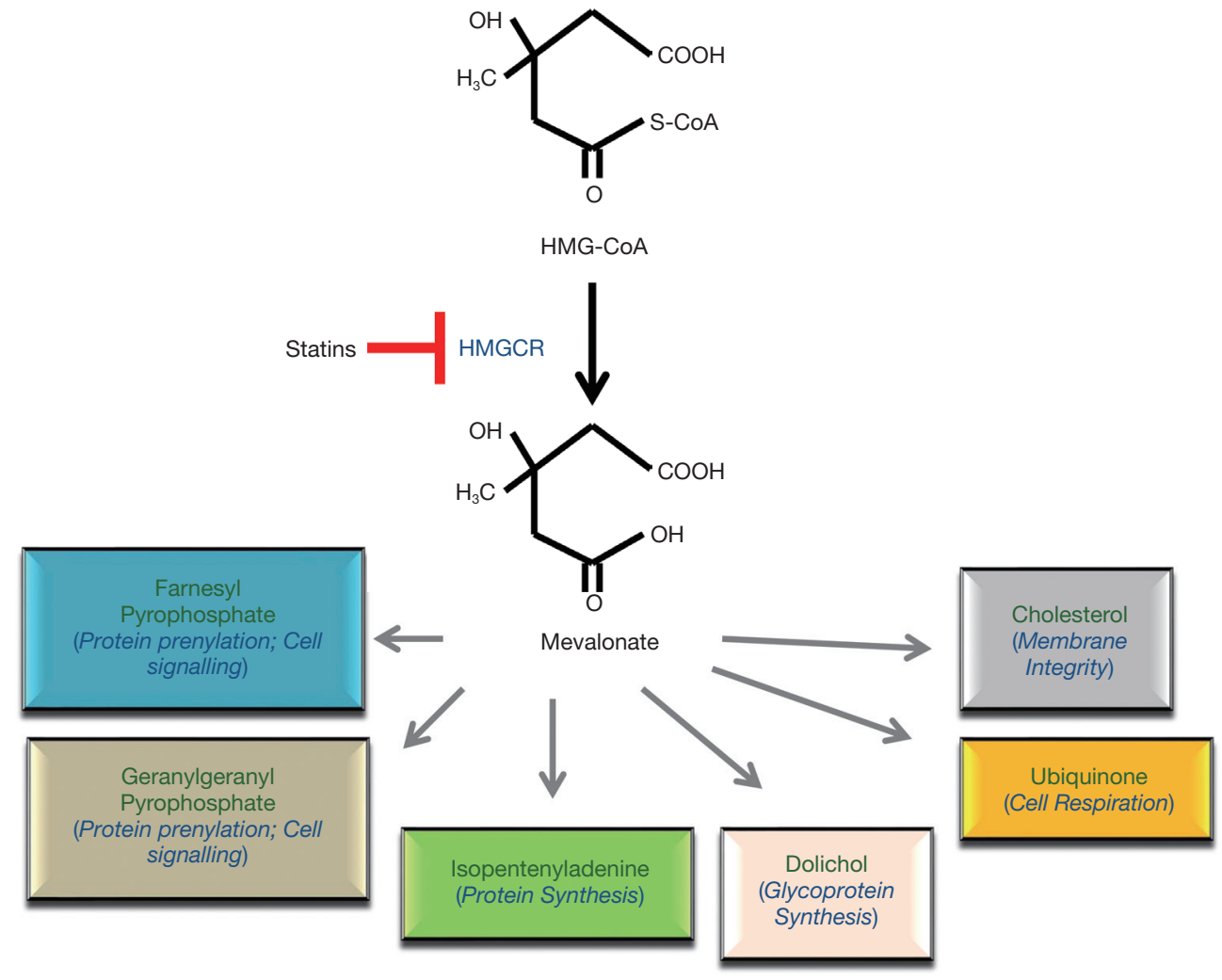

Figure 1 Mevalonate pathway and its downstream products with their main function in brackets.

much of the apoptotic machinery and pathways remain intact in tumours, making them attractive targets for therapeutic intervention (3).

Statins are among drugs that have been shown to possess apoptosis-inducing effects. They were originally developed as a treatment for hypercholesterolemia in the 1970s, and shortly after the discovery of mevastatin, its analogues including simvastatin, lovastatin, and pravastatin were developed (4). Statins target and inhibit 3-hydroxy3-methylglutaryl-CoA reductase (HMGCR), the highly regulated rate-limiting enzyme in the mevalonate (MVA) pathway (Figure 1). Statins can be classified as natural or fungal-derived (lovastatin, simvastatin, pravastatin), and synthetic (fluvastatin, atorvastatin, rosuvastatin, pitavastatin, and cerivastatin). The two groups differ in their ability to inhibit HMGCR and in their lipophilicity. Among these agents, lovastatin, simvastatin, atorvastatin, and fluvastatin are lipophilic, whereas pravastatin and rosuvastatin are more hydrophilic. The pharmacological activity of statins is dictated by their different chemical structures, lipophilicity/ hydrophilicity, kinetic profile, rate of metabolism, and the formation of active and inactive metabolites (5).
The most severe side effect of statins is myotoxicity and rhabdomyolysis, which appears to be associated with the presence of co-existing conditions, including hepatic insufficiency, cholestasis or renal diseases (6).

In cancer patients, the efficacy of statins as anticancer agents has been evaluated both in monotherapy and in combination therapy with currently used chemotherapeutics (5). Many studies have also shown that statins induce programmed cell death in a subset of cell lines derived from tumours in tissue culture, implying that the corresponding cancers could be sensitive to statin-specific apoptosis in vivo (7-9). The objectives of this article are two-fold: (I) to review the latest preclinical and clinical literature focused on the potential utility of statins as anticancer drugs; and (II) to discuss the molecular mechanisms driving their effects.

\section{Observations from clinical studies}

The efficacy of statins as anticancer agents has been evaluated both in monotherapy and in combination therapy with currently used chemotherapeutic drugs. To a varying degree of success, studies have shown the potential 
mortality benefits of statin consumption in patients with different types of cancers, which include esophageal, breast, lung, liver, pancreatic, endometrial, and colorectal cancer (10-20).

In a randomized control trial by Kawata and colleagues, the efficacy of pravastatin in chemotherapy was tested in patients with unresectable hepatocellular carcinoma (21). Results showed that pravastatin slowly, but significantly, reduced the diameter of the main hepatic lesions 1 year after the start of the treatment. Complete remission was obtained in $73 \%(11 / 15)$ of new patients and in $41 \%(9 / 22)$ of salvage patients (22). A retrospective cohort study was conducted on data obtained from Central Cancer Registry on 15,422 patients who were diagnosed with hepatocellular carcinoma between 2002 and 2016. The study concluded that post-diagnosis statin use was associated with reduced mortality in hepatocellular carcinoma (23).

A large population-based cohort of 12,572 patients ages 65 years or older supports potential benefits of statins on improving survival among elderly patients with primary pancreatic ductal adenocarcinoma (24). Another recent meta-analysis of 26 studies performed on more than 3 million participants and 170,000 pancreatic cancer patients suggested that statins might have a preventive effect on pancreatic cancer. However, authors of this article believed that more high-quality randomized clinical trials and cohort studies should be carried out to arrive at a convincing conclusion regarding this protective effect of statins against pancreatic cancer (12).

A phase I clinical trial showed the safety and overall positive response of adding pravastatin to idarubicin and high-dose cytarabine in patients with acute myelogenous leukemia (AML) (22). Furthermore, a number of Phase II randomized control trials were specifically designed to answer questions such as effect of atorvastatin versus placebo for lowering mammography-defined breast density and other surrogate markers associated with breast cancer risk; the effects of atorvastatin on tumour proliferation in postmenopausal women undergoing treatment for breast cancer; and fluvastatin's effect on biomarkers in women undergoing surgery for ductal carcinoma in situ or stage I breast cancer (25-28). A Swedish nation-wide retrospective cohort study of 20,559 Swedish women diagnosed with breast cancer between July 2005 through 2008 revealed that compared to non- or irregular use, regular pre-diagnostic statin use was associated with lower risk of breast cancer related deaths. The same study also showed that even post- diagnostic statin use compared to non-use was associated with lower risk of breast cancer-related deaths (29).

A retrospective population-based study concluded that statin use has been associated with superior survival in patients suffering from ovarian cancer (16). In an ongoing study patients have been recruited for a Phase II trial to study the synergistic interaction between lovastatin and paclitaxel for women with refractory or relapsed ovarian cancer. In a recent clinical trial, it was found that in patients with prostate cancer treated with a moderate hypofractionated intensity-modulated radiotherapy schedule, use of statins lowered the incidence and grade of acute rectal toxicity (30). A Finnish nationwide cohort study revealed that patients taking statins had significantly lower risk of starting androgen deprivation therapy and decreased mortality rate related to prostate cancer. This study concluded that the risk was lower especially among men with statin use before prostate cancer diagnosis and in men who used statins at high-dose (31). Although accumulating evidence supports statins' radiosensitizing properties and their beneficial antitumor effects in prostate cancer, more clinical trials are warranted prior to the routine implementation of statins in treatment regimes.

In a propensity-matched study, it was shown that among patients who were suffering from non-small cell lung cancer, statin administration was associated with positive outcomes (32). These included cancer characteristics, staging work-up and chemotherapy use. An observational study reported by Omori indicated that non-small cell lung cancer patients who have been previously treated with nivolumab had an increased response rate and longer time-to-treatment failure if prescribed statins. However, this response was not statistically significant for overall survival (33). A meta-analysis of observational studies concluded that statin-administered patients suffering from lung cancer had significantly improved overall survival (34). Subgroup analyses performed in this study revealed that statin users were more probable to have better survival rate in stage IV lung cancer patients (HR 0.77, 95\% CI: 0.74-0.79) than in mixed stage (I-IV or I-III) patients (34). Khurana et al. conducted a large case controlled study of a veteran population with almost 200 lung cancer cases among statin users and found a $45 \%$ reduction in lung cancer risk among statin users when compared to non-users (35). Farwell and colleagues reported a 30\% reduction in lung cancer risk among statin users in a retrospective cohort study of another veteran population (36). 


\section{Insights from preclinical studies}

\section{Molecular mechanisms}

Understanding the exact mechanisms underlying statininduced apoptosis is an emerging topic in cancer research that requires further investigation. Nonetheless, it has been shown that statins induce apoptosis in sensitive cells directly via the inhibition of HMGCR activity. It is the biologically active, open-ring structure of each statin that blocks HMGCR activity to trigger apoptosis (37). Moreover, cells have been made statin-resistant after being cultured in the presence of increasing statin concentrations by amplifying the gene for HMGCR (38). In addition, when cells sensitive to statin-induced apoptosis were coincubated with statins and mevalonate, the direct product of HMGCR activity, apoptosis was inhibited (39). In other studies, statin-sensitive cells were co-incubated with each mevalonate end product and a statin to ascertain which molecules in the pathway could prevent statin-induced apoptosis. Interestingly, most reports indicate that only geranylgeranyl pyrophosphate (GGPP) is able to inhibit statin-induced apoptosis (39). Based on these observations, it is believed that upon statin treatment, cellular GGPP levels are depleted, resulting in deficient isoprenylation of proteins upon which tumour cells are dependent upon. This, in turn, would lead to mislocalization and malfunction of such proteins, ultimately causing the tumour cell to undergo apoptosis.

\section{The MVA patbway and HMGCR regulation}

HMGCR is tightly regulated at transcriptional, translational and post-translational levels. Inhibition of HMGCR with statins deprives the cell of the end products of the MVA pathway, and results in a feedback response that leads to the upregulation of the MVA pathway (40). The protein level and activity of HMGCR is also regulated by phosphorylation and degradation. Mechanistically, the increase in the rate of HMGCR degradation when sterol concentrations are high was studied using a number of either mutant or deletion forms of HMGCR $(41,42)$. These studies highlighted that the element responsible for the regulated degradation of HMGCR resides in the transmembrane region, and in contrast, the degradation of the cytoplasmic portion of the protein is not influenced by sterols. Furthermore, the kinase that regulates HMGCR activity is AMPK, which phosphorylates serine-872, and results in reduced enzymatic activity.

A growing number of studies suggest that the MVA pathway plays a significant role in the regulation of cellular proliferation and transformation. Overall, it has been established that statin treatment of hypercholesterolemia results in depletion of the MVA intermediates, including farnesyl pyrophosphate (FPP) and GGPP, which are required for proper function of small GTPases. Given many Ras proteins are prevalently mutated in pancreatic cancer, attempts have been made to examine the effect of three key intermediates of the MVA pathway on GFP-KRas protein localization and the gene expression profile in pancreatic cancer cells after exposure to individual statins by whole genome DNA microarray analysis. Based on some recent observations, it has been suggested that the anticancer effects of statins most likely were mediated through isoprenoid intermediates of the MVA pathway, as they influenced expression of genes involved in multiple intracellular pathways $(8,37,43)$. Figure 2 summarizes potential mechanisms of HMGCR dysregulation in cancer.

\section{Liver kinase B1 (LKB1) and AMPK involvement}

The LKB1, encoded by the tumour suppressor gene STK11, is a serine/threonine kinase which harbors germ-line mutations in Peutz-Jeghers syndrome (PJS), an inherited cancer predisposition, and somatic mutations in sporadic cancers (44). By phosphorylating several key cellular kinases, LKB1 regulates cellular metabolism; cell polarity; and a variety of other functions ranging from proliferation and migration to senescence, apoptosis, DNA damage response and differentiation (45). LKB1 has been shown to be the major upstream kinase and activator of 5 ' adenosine monophosphate-activated protein kinase (AMPK) $(46,47)$. On the other hand, the kinase that has been shown most to regulate HMGCR activity is AMPK, which phosphorylates serine-872 (S872), and results in reduced HMGCR enzymatic activity $(48,49)$.

A combination therapy of mevastatin and LBH589, a histone deacetylase inhibitor (HDACi) was shown to inhibit autophagic flux by preventing Vps34/Beclin 1 complex formation and downregulating prenylated Rab7, an active form of the small GTPase necessary for autophagosomelysosome fusion. Mevastatin also was reported to increase HDACi LBH589-induced cell death in triple-negative breast cancer (TNBC) cells suggesting co-treatment with mevastatin and LBH589 activated LKB1/AMPK signaling 


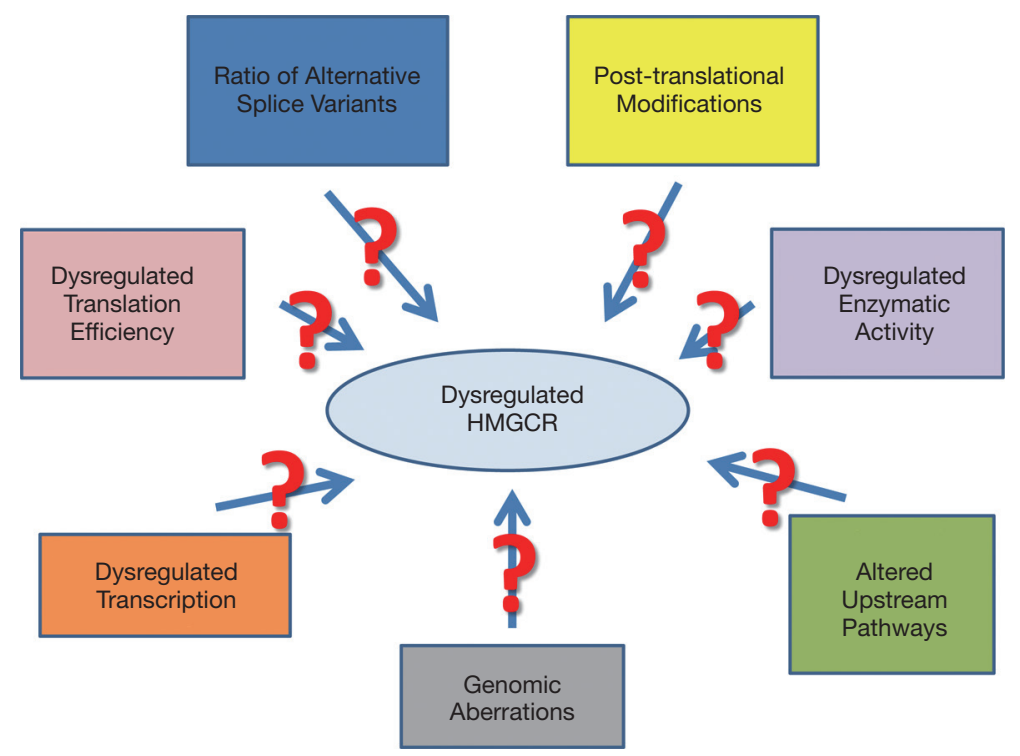

Figure 2 Potential mechanisms of HMGCR dysregulation in cancer. HMGCR, 3-hydroxy-3-methylglutaryl-CoA reductase.

and subsequently inhibited mTOR (50).

\section{Other putative pathways}

Several other putative mechanisms have been proposed to play an important role in driving the observed anticancer effects of statins. The synergistic effect of rosuvastatin with dasatinib against hepatocellular carcinoma is believed to be mediated by a decrease in the p-FAK/p-Src, p-Ras/ c-Raf, p-STAT-3, and p-Akt levels which could result in an enhancement of apoptosis by an increase in caspase-3 level and a decline in survivin level (51). In a study investigating the effects of simvastatin treatment on hepatocellular carcinoma, it was found that simvastatin induces G0/G1 arrest by upregulating $\mathrm{p} 21$ and $\mathrm{p} 27$ by activating AMPK and inhibiting the STAT3-Skp2 axis, respectively (52). Simvastatin also induced caspase-dependent apoptosis in A549 lung cancer cells, where it was associated with a decrease in the expression of phosphorylated Akt and downregulated survivin mRNA and protein (53).

As Wang and colleagues showed, atorvastatin and caffeine in combination cause down-regulation of phospho-Akt, phospho-Erk1/2, anti-apoptotic Bcl-2 and survivin protein levels in prostate cancer cells lines. This is suggestive that such a combination therapy may be effective in inhibiting the growth of prostate cancer (54). In another study on prostate cancer, using microarrays, it was shown that simvastatin treatment resulted in up- regulation of annexin A10 (ANXA10) in PC-3 cells. ANXA10 is believed to have antitumor effects (55). Statins have also been shown to affect the Rho signalling pathway in inducing tumour-specific apoptosis (56). Both lovastatin and simvastatin induced activation of caspase-8, caspase-3, and, to a lesser extent, caspase-9. Additionally, both drugs suppressed expression of $\mathrm{Rb}$, phosphorylated $\mathrm{Rb}$, cyclin $\mathrm{D} 1$, cyclin D3, CDK4, and CDK6, but induced p21 and p27 expression in prostate cancer cells. Furthermore, lovastatin and simvastatin suppressed RhoA activation and c-JUN expression, but not cyclooxygenase-2 expression (56). This data suggests that the underlying molecular mechanism of statins' action is mediated through inactivation of RhoA, which in turn induces caspase enzymatic activity and/or G(1) cell cycle.

Research done by Tsubaki et al. found that the sensitivity of head and neck carcinoma cells to statins is related to the expression of their Ras expression status, and statin-induced apoptosis is mediated via suppression of the Ras/ERK and Ras/mTOR pathways (57). Statin-induced apoptosis and the Ras signalling pathway have also been implicated in human hematopoietic tumour cells (58). Fujiwara and colleagues found that statins induce apoptosis by decreasing the mitochondrial transmembrane potential, increasing the activation of caspase-9 and caspase-3, enhancing Bim expression, and inducing cell-cycle arrest at G1 phase through inhibition of Ras/extracellular signalregulated kinase and Ras/mammalian target of rapamycin 
pathways (58).

In a study considering glioblastoma cell lines, it was shown that statins induced apoptosis via the suppression of ERK1/2 and Akt activation through inhibition of GGPP biosynthesis. The authors also observed an increase in caspase- 3 activity. The apoptosis induced by statins was not inhibited by the addition of FPP, squalene, ubiquinone, and isopentenyladenine, but by GGPP (59). Increased caspase activity and depolarization of the mitochondrial membrane secondary to mevastatin exposure was also noted in myeloma cells. In this study, expression of Bcl-2 mRNA and protein was down-regulated, with no change in Bax or Bcl-XL protein production (60).

Another study suggested that the antiproliferative effects of simvastatin on murine melanoma cells were mediated mainly via suppression of the heterodimeric transcription factor hypoxia-inducible factor (HIF-1 $\alpha$ ) (61). Simvastatin may act as a heat shock protein 90 (Hsp90) inhibitor to prevent the formation of the K292-acetylated Hsp90/ Cdc37 complex in TNBC cells (62). Recent work focusing on osteosarcoma cell lines indicated that simvastatin significantly induced apoptosis, increased the Bax/Bcl-2 ratio, and cleavage of caspase-3 (63).

Finally, as Martirosyan and colleagues showed, lovastatin drives ovarian tumor cell death by two mechanisms: first, by blocking HMG-CoA reductase activity, and second, by sensitizing multi-drug resistant cells to doxorubicin by a novel mevalonate-independent mechanism. This inhibition of drug transport, likely through inhibition of P-glycoprotein, potentiates both DNA damage and tumor cell apoptosis (64).

\section{Conclusions}

Numerous studies have shown that statins have anticancer benefits in many tumour types. In breast and colorectal cancers, there are epidemiological studies suggesting an association between statin use and lower cancer risk. Although many studies have shown promising benefits for using statins among cancer patients, there is still a need for a better understanding of the molecular determinants of statin-sensitivity in various cancer types to advance personalized medicine and to develop biomarkers of statinsensitive tumours.

\section{Acknowledgments}

None.

\section{Footnote}

Conflicts of Interest: The author has no conflicts of interest to declare.

Ethical Statement: The author is accountable for all aspects of the work in ensuring that questions related to the accuracy or integrity of any part of the work are appropriately investigated and resolved.

\section{References}

1. Siegel RL, Miller KD, Jemal A. Cancer statistics, 2019. CA Cancer J Clin 2019;69:7-34.

2. Wong RS. Apoptosis in cancer: from pathogenesis to treatment. J Exp Clin Cancer Res 2011;30:87.

3. Zimmermann KC, Bonzon C, Green DR. The machinery of programmed cell death. Pharmacol Ther 2001;92:57-70.

4. Tobert JA. New developments in lipid-lowering therapy: the role of inhibitors of hydroxymethylglutaryl-coenzyme A reductase. Circulation 1987;76:534-8.

5. Gazzerro P, Proto MC, Gangemi G, et al. Pharmacological actions of statins: a critical appraisal in the management of cancer. Pharmacol Rev 2012;64:102-46.

6. Bellosta S, Paoletti R, Corsini A. Safety of statins: focus on clinical pharmacokinetics and drug interactions. Circulation 2004;109:III50-7.

7. Paškevičiūtè $M$, Petrikaite $V$. Differences of statin activity in $2 \mathrm{D}$ and $3 \mathrm{D}$ pancreatic cancer cell cultures. Drug Des Devel Ther 2017;11:3273-80.

8. Wong WW, Clendening JW, Martirosyan A, et al. Determinants of sensitivity to lovastatin-induced apoptosis in multiple myeloma. Mol Cancer Ther 2007;6:1886-97.

9. Vaklavas C, Chatzizisis YS, Tsimberidou AM. Common cardiovascular medications in cancer therapeutics. Pharmacol Ther 2011;130:177-90.

10. Lacroix O, Couttenier A, Vaes E, et al. Statin use after diagnosis is associated with an increased survival in esophageal cancer patients: a Belgian population-based study. Cancer Causes Control 2019;30:385-93.

11. Xia DK, Hu ZG, Tian YF, et al. Statin use and prognosis of lung cancer: a systematic review and meta-analysis of observational studies and randomized controlled trials. Drug Des Devel Ther 2019;13:405-22.

12. Zhang Y, Liang $M$, Sun $C$, et al. Statin Use and Risk of Pancreatic Cancer: An Updated Meta-analysis of 26 Studies. Pancreas 2019;48:142-50.

13. Langballe R, Cronin-Fenton D, Dehlendorff C, et 
al. Statin use and risk of contralateral breast cancer: a nationwide cohort study. Br J Cancer 2018;119:1297-305.

14. Nguyen T, Khan A, Liu Y, et al. The Association Between Statin Use After Diagnosis and Mortality Risk in Patients With Esophageal Cancer: A Retrospective Cohort Study of United States Veterans. Am J Gastroenterol 2018;113:1310.

15. Sperling CD, Verdoodt F, Kjaer Hansen M, et al. Statin use and mortality among endometrial cancer patients: a Danish nationwide cohort study. Int J Cancer 2018;143:2668-76.

16. Couttenier A, Lacroix O, Vaes E, et al. Statin use is associated with improved survival in ovarian cancer: A retrospective population-based study. PLoS One 2017;12:e0189233.

17. Mei Z, Liang M, Li L, et al. Effects of statins on cancer mortality and progression: A systematic review and metaanalysis of 95 cohorts including 1,111,407 individuals. Int J Cancer 2017;140:1068-81.

18. Cardwell CR, Mc Menamin U, Hughes CM, et al. Statin use and survival from lung cancer: a populationbased cohort study. Cancer Epidemiol Biomarkers Prev 2015;24:833-41.

19. Cardwell CR, Hicks BM, Hughes C, et al. Statin use after colorectal cancer diagnosis and survival: a populationbased cohort study. J Clin Oncol 2014;32:3177-83.

20. Boudreau DM, Yu O, Johnson J. Statin use and cancer risk: a comprehensive review. Expert Opin Drug Saf 2010;9:603-21.

21. Kawata S, Yamasaki E, Nagase T, et al. Effect of pravastatin on survival in patients with advanced hepatocellular carcinoma. A randomized controlled trial. Br J Cancer 2001;84:886-91.

22. Kornblau SM, Banker DE, Stirewalt D, et al. Blockade of adaptive defensive changes in cholesterol uptake and synthesis in AML by the addition of pravastatin to idarubicin + high-dose Ara-C: a phase 1 study. Blood 2007;109:2999-3006.

23. Thrift AP, Natarajan Y, Liu Y, et al. Statin Use After Diagnosis of Hepatocellular Carcinoma Is Associated With Decreased Mortality. Clin Gastroenterol Hepatol 2019;17:2117-25.e3.

24. e JY, Lu SE, Lin Y, et al. Differential and Joint Effects of Metformin and Statins on Overall Survival of Elderly Patients with Pancreatic Adenocarcinoma: A Large Population-Based Study. Cancer Epidemiol Biomarkers Prev 2017;26:1225-32.

25. Beckwitt CH, Brufsky A, Oltvai ZN, et al. Statin drugs to reduce breast cancer recurrence and mortality. Breast
Cancer Res 2018;20:144.

26. Borgquist S, Bjarnadottir O, Kimbung S, et al. Statins: a role in breast cancer therapy? J Intern Med 2018;284:346-57.

27. Ji Y, Rounds T, Crocker A, et al. The Effect of Atorvastatin on Breast Cancer Biomarkers in High-Risk Women. Cancer Prev Res (Phila) 2016;9:379-84.

28. Arun BK, Gong Y, Liu D, et al. Phase I biomarker modulation study of atorvastatin in women at increased risk for breast cancer. Breast Cancer Res Treat 2016;158:67-77.

29. Borgquist S, Broberg P, Tojjar J, et al. Statin use and breast cancer survival - a Swedish nationwide study. BMC Cancer 2019;19:54.

30. Hutchinson J, Marignol L. Clinical Potential of Statins in Prostate Cancer Radiation Therapy. Anticancer Res 2017;37:5363-72.

31. Joentausta RM, Rannikko A, Murtola TJ. Prostate cancer survival among statin users after prostatectomy in a Finnish nationwide cohort. Prostate 2019;79:583-91.

32. Lin JJ, Ezer N, Sigel K, et al. The effect of statins on survival in patients with stage IV lung cancer. Lung Cancer 2016;99:137-42.

33. Omori M, Okuma Y, Hakozaki T, et al. Statins improve survival in patients previously treated with nivolumab for advanced non-small cell lung cancer: An observational study. Mol Clin Oncol 2019;10:137-43.

34. Chen Y, Li X, Zhang R, et al. Effects of statin exposure and lung cancer survival: A meta-analysis of observational studies. Pharmacol Res 2019;141:357-65.

35. Khurana V, Bejjanki HR, Caldito G, et al. Statins reduce the risk of lung cancer in humans: a large case-control study of US veterans. Chest 2007;131:1282-8.

36. Farwell WR, Scranton RE, Lawler EV, et al. The association between statins and cancer incidence in a veterans population. J Natl Cancer Inst 2008;100:134-9.

37. Wong WW, Tan MM, Xia Z, et al. Cerivastatin triggers tumor-specific apoptosis with higher efficacy than lovastatin. Clin Cancer Res 2001;7:2067-75.

38. Ravid T, Avner R, Polak-Charcon S, et al. Impaired regulation of 3-hydroxy-3-methylglutaryl-coenzyme A reductase degradation in lovastatin-resistant cells. J Biol Chem 1999;274:29341-51.

39. Xia Z, Tan MM, Wong WW, et al. Blocking protein geranylgeranylation is essential for lovastatin-induced apoptosis of human acute myeloid leukemia cells. Leukemia 2001;15:1398-407.

40. Goldstein JL, Brown MS. Regulation of the mevalonate pathway. Nature 1990;343:425-30. 
41. Gil G, Faust JR, Chin DJ, et al. Membrane-bound domain of HMG CoA reductase is required for sterol-enhanced degradation of the enzyme. Cell 1985;41:249-58.

42. Roitelman J, Bar-Nun S, Inoue S, et al. Involvement of calcium in the mevalonate-accelerated degradation of 3-hydroxy-3-methylglutaryl-CoA reductase. J Biol Chem 1991;266:16085-91.

43. Gbelcová H, Rimpelova S, Knejzlik Z, et al. Isoprenoids responsible for protein prenylation modulate the biological effects of statins on pancreatic cancer cells. Lipids Health Dis 2017;16:250.

44. Hezel AF, Bardeesy N. LKB1; linking cell structure and tumor suppression. Oncogene 2008;27:6908-19.

45. Vaahtomeri K, Makela TP. Molecular mechanisms of tumor suppression by LKB1. FEBS Lett 2011;585:944-51.

46. Woods A, Johnstone SR, Dickerson K, et al. LKB1 is the upstream kinase in the AMP-activated protein kinase cascade. Curr Biol 2003;13:2004-8.

47. Shaw RJ, Bardeesy N, Manning BD, et al. The LKB1 tumor suppressor negatively regulates mTOR signaling. Cancer Cell 2004;6:91-9.

48. Clarke PR, Hardie DG. Regulation of HMG-CoA reductase: identification of the site phosphorylated by the AMP-activated protein kinase in vitro and in intact rat liver. EMBO J 1990;9:2439-46.

49. Omkumar RV, Darnay BG, Rodwell VW. Modulation of Syrian hamster 3-hydroxy-3-methylglutaryl-CoA reductase activity by phosphorylation. Role of serine 871. J Biol Chem 1994;269:6810-4.

50. Lin Z, Zhang Z, Jiang X, et al. Mevastatin blockade of autolysosome maturation stimulates LBH589-induced cell death in triple-negative breast cancer cells. Oncotarget 2017;8:17833-48.

51. El Sayed I, Helmy MW, El-Abhar HS. Inhibition of SRC/FAK cue: A novel pathway for the synergistic effect of rosuvastatin on the anti-cancer effect of dasatinib in hepatocellular carcinoma. Life Sci 2018;213:248-57.

52. Wang ST, Ho HJ, Lin JT, et al. Simvastatin-induced cell cycle arrest through inhibition of STAT3/SKP2 axis and activation of AMPK to promote $\mathrm{p} 27$ and $\mathrm{p} 21$ accumulation in hepatocellular carcinoma cells. Cell Death Dis 2017;8:e2626.

53. Hwang KE, Na KS, Park DS, et al. Apoptotic induction by simvastatin in human lung cancer A549 cells via Akt signaling dependent down-regulation of survivin. Invest New Drugs 2011;29:945-52.

54. Wang Z, Zhang L, Wan Z, et al. Atorvastatin and Caffeine in Combination Regulates Apoptosis, Migration, Invasion and Tumorspheres of Prostate Cancer Cells. Pathol Oncol Res 2018. [Epub ahead of print].

55. Miyazawa Y, Sekine Y, Kato H, et al. Simvastatin UpRegulates Annexin A10 That Can Inhibit the Proliferation, Migration, and Invasion in Androgen-Independent Human Prostate Cancer Cells. Prostate 2017;77:337-49.

56. Hoque A, Chen $\mathrm{H}, \mathrm{Xu} \mathrm{XC}$. Statin induces apoptosis and cell growth arrest in prostate cancer cells. Cancer Epidemiol Biomarkers Prev 2008;17:88-94.

57. Tsubaki M, Fujiwara D, Takeda T, et al. The sensitivity of head and neck carcinoma cells to statins is related to the expression of their Ras expression status, and statininduced apoptosis is mediated via suppression of the Ras/ ERK and Ras/mTOR pathways. Clin Exp Pharmacol Physiol 2017;44:222-34.

58. Fujiwara D, Tsubaki M, Takeda T, et al. Statins induce apoptosis through inhibition of Ras signaling pathways and enhancement of Bim and p27 expression in human hematopoietic tumor cells. Tumour Biol 2017;39:1010428317734947.

59. Yanae M, Tsubaki M, Satou T, et al. Statin-induced apoptosis via the suppression of ERK1/2 and Akt activation by inhibition of the geranylgeranyl-pyrophosphate biosynthesis in glioblastoma. J Exp Clin Cancer Res 2011;30:74.

60. Jánosi J, Sebestyen A, Bocsi J, et al. Mevastatin-induced apoptosis and growth suppression in U266 myeloma cells. Anticancer Res 2004;24:1817-22.

61. Licarete E, Sesarman A, Rauca VF, et al. HIF-1alpha acts as a molecular target for simvastatin cytotoxicity in B16. F10 melanoma cells cultured under chemically induced hypoxia. Oncol Lett 2017;13:3942-50.

62. Kou X, Jiang X, Liu H, et al. Simvastatin functions as a heat shock protein 90 inhibitor against triple-negative breast cancer. Cancer Sci 2018;109:3272-84.

63. Kany S, Woschek M, Kneip N, et al. Simvastatin exerts anticancer effects in osteosarcoma cell lines via geranylgeranylation and c-Jun activation. Int J Oncol 2018;52:1285-94.

64. Martirosyan A, Clendening JW, Goard CA, et al. Lovastatin induces apoptosis of ovarian cancer cells and synergizes with doxorubicin: potential therapeutic relevance. BMC Cancer 2010;10:103.

Cite this article as: Fatehi Hassanabad A. Current perspectives on statins as potential anti-cancer therapeutics: clinical outcomes and underlying molecular mechanisms. Transl Lung Cancer Res 2019;8(5):692-699. doi: 10.21037/tlcr.2019.09.08 